\title{
Approximate Analytical Solution of Nonlinear Reaction's Diffusion Equation at Conducting Polymer Ultramicroelectrodes
}

\author{
Anitha Shanmugarajan, ${ }^{1}$ Subbiah Alwarappan, ${ }^{2}$ and Rajendran Lakshmanan ${ }^{1}$ \\ ${ }^{1}$ Department of Mathematics, The Madura College, Madurai-625011, India \\ ${ }^{2}$ Nanomaterials Research and Education Center, University of South Florida, Tampa, FL, USA
}

Correspondence should be addressed to Rajendran Lakshmanan, raj_sms@rediffmail.com

Received 14 November 2011; Accepted 22 December 2011

Academic Editors: R. Gómez and H. Luo

Copyright (C) 2012 Anitha Shanmugarajan et al. This is an open access article distributed under the Creative Commons Attribution License, which permits unrestricted use, distribution, and reproduction in any medium, provided the original work is properly cited.

\begin{abstract}
A theoretical model of reaction/diffusion within conducting polymer microelectrodes is discussed. The model is based on the steady-state diffusion equation containing a nonlinear term related to the Michaelis-Menten kinetic of the enzymatic reaction. An analytical expression pertaining to the concentration of substrate and current is obtained using homotopy perturbation method for all values of diffusion and the saturation parameter. The substrate concentration profile and current response can be used in a large range of concentrations including the non-linear contributions. These approximate analytical results were found to be in good agreement with the previously reported limiting case results.
\end{abstract}

\section{Introduction}

The advantages of the ultramicroelectrodes (UMEs) include steady-state current, rapid response time, minimal iR drop, lower detection limits, and sensitive analysis in a highly resistive medium [1-4]. Furthermore, due to their diminished surface area or radii of the electrodes, they are often employed as probes to monitor various chemical events occurring inside the living cell [5-8]. Ultramicroelectrodes possess many advantages for studying electrochemical kinetics and in electroanalytical applications, imaging, and surface modification [9]. Microelectrodes modified with a polymer film find potential application in various sensing applications [10-12]. The working principle of polymer-modified ultramicroelectrodes occurs in the following manner: initially, the redox analyte interacts with the immobilized active receptor sites present in the polymer matrix, then at the underlying electrode surface. Briefly, we can say that the redox reaction is mediated by the polymeric layer. Further, due to the electroactive property of the polymer, charge can percolate through the polymer chain and thereby reaches the electrode/interface to give rise to a redox current and is directly proportional to the concentration of the analyte. The electron transfer occurs between the substrate and the catalytic receptor site and as a result the kinetics of the substrate/product transformation will be governed by the properties of the mediating electroactive polymer film.

Recent advancements in ultramicroelectrodes modified with conducting polymers were reported elsewhere [1318]. Further, the analytical applications of various polymermodified sensors and the reaction/diffusion at the conducting polymer electrode (where the chemical reaction term is described by Michaelis-Menten kinetics) were reviewed extensively by various groups [19-23]. Recently, Lyons et al. [24] evaluated the analytical solutions corresponding to the steady-state substrate concentration profile and current observed at a conducting polymer microelectrode when the substrate concentration is low. For higher values of the substrate concentration, a kinetic rate law based on the Michaelis-Menten equation is more appropriate. Recently, Anitha et al. [25] derived the analytical expression for nonsteady-state concentrations of substrate and mediator at a polymer modified ultramicroelectrodes using reduction of order method. However, to the best of our knowledge, there were no analytical results available till date that corresponds to the steady-state substrate concentration and current for all possible values of diffusion parameter $\gamma$ and the saturation parameter $\alpha$. However, in general, analytical solutions of 


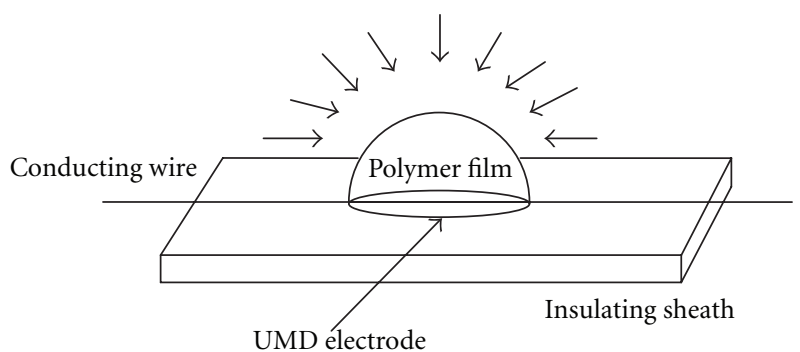

FIGURE 1: Schematic representations of the geometry adopted by the polymer-coated microelectrode and the expected diffusion profile adopted by the analytes.

TABLE 1: Approximate expressions of concentration of substrate and current under the boundary conditions (2a) and (2b).

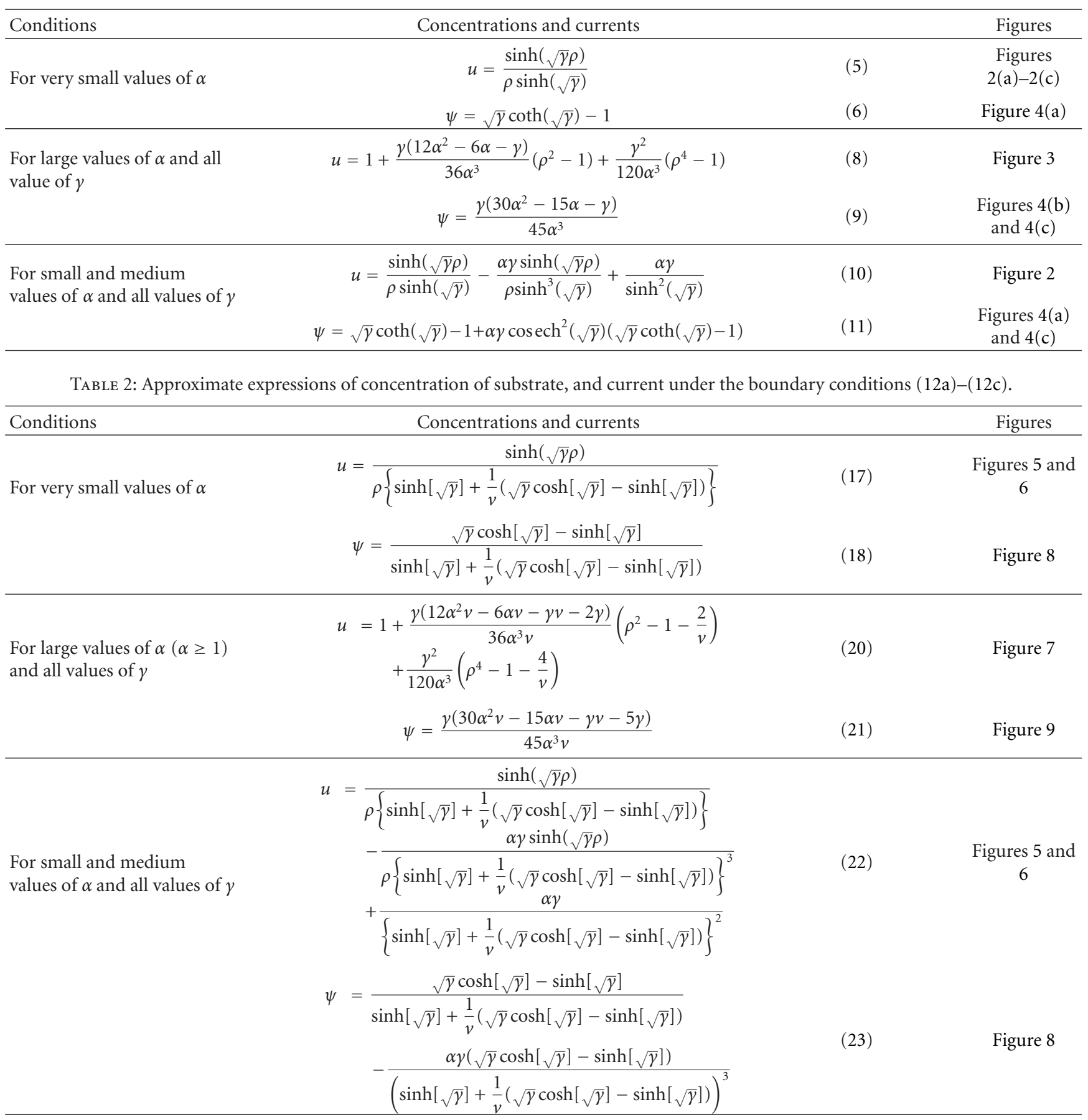




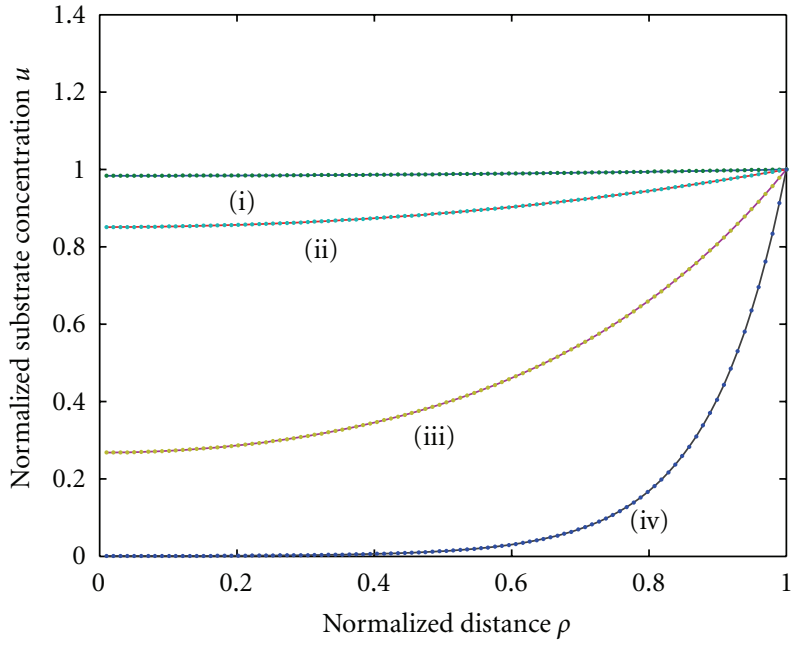

(a)

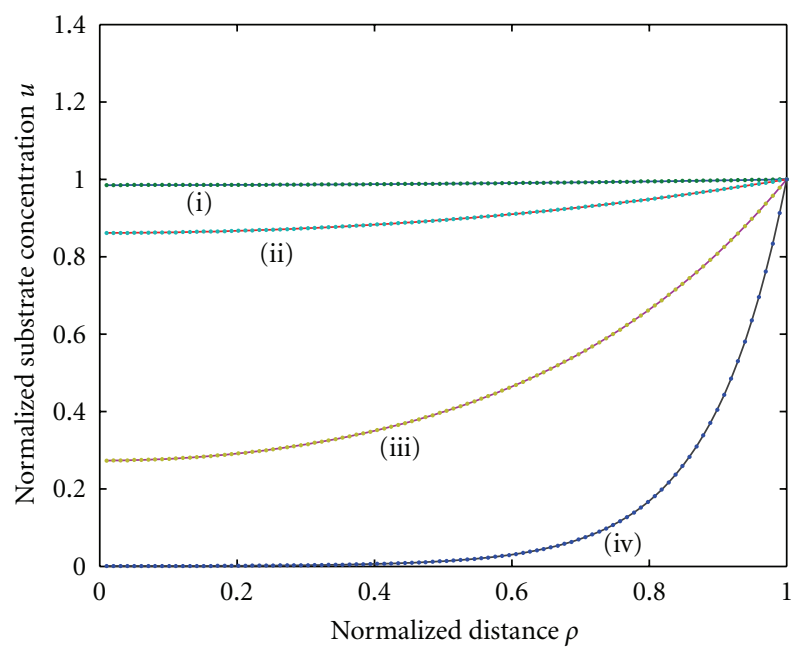

(c)

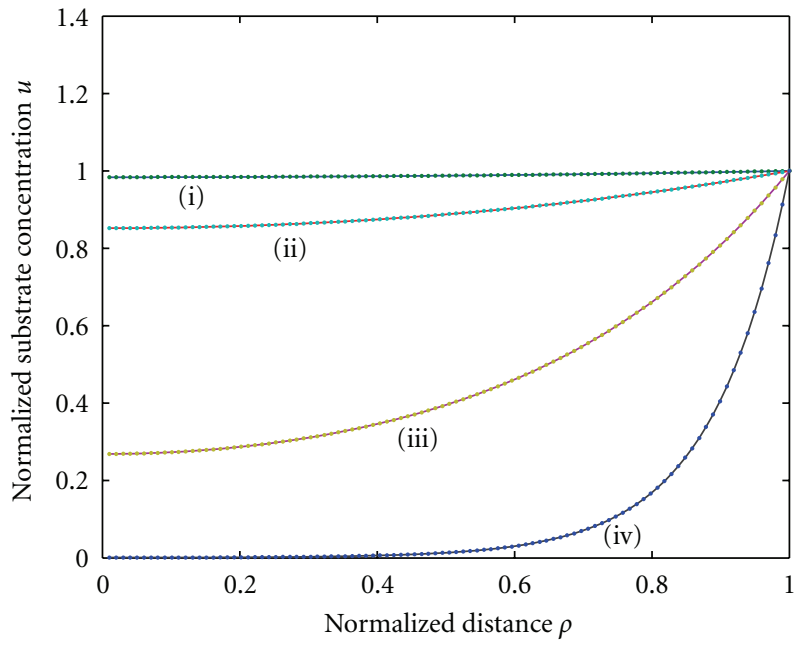

(b)

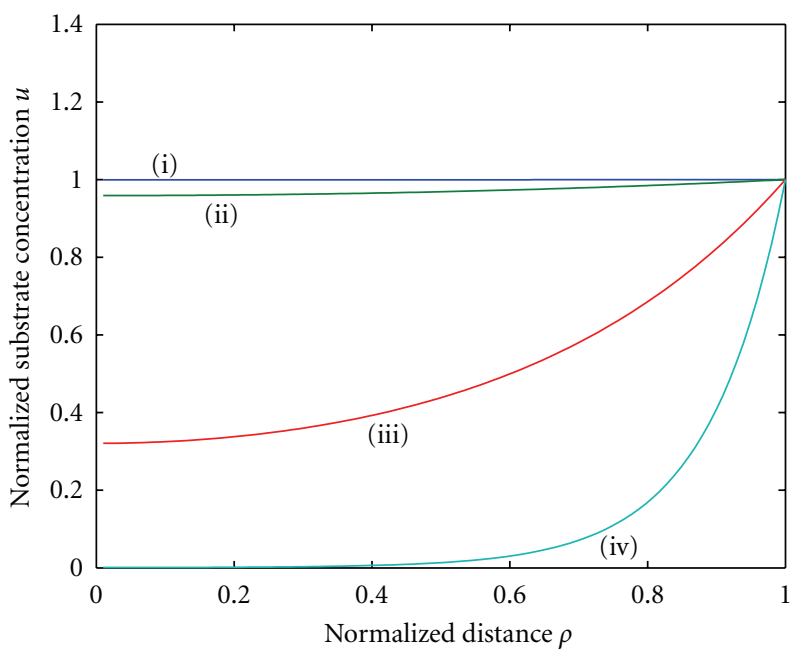

(d)

Figure 2: Plot of normalized substrate concentration " $u$ " at a microelectrode modified with a polymer film as a function of " $\rho$ ". The concentrations were computed for various values of the reaction diffusion parameter " $\gamma$ " when (a) $\alpha=0.001$, (b) $\alpha=0.01$, (c) $\alpha=0.1$, and (d) $\alpha=1$. (solid line) (10); (dotted line) (5). Solid lines are compared with points. In this figure (i) $\gamma=0.1$, (ii) $\gamma=1$, (iii) $\gamma=10$, and (iv) $\gamma=100$.

nonlinear differential equations are more interesting and useful numerical solutions, as they are used in various kinds of data analysis. Therefore, herein, we employ analytical method to evaluate the steady-state substrate concentration and current for all possible values of diffusion and saturation parameter.

\section{Mathematical Formulation of the Boundary Value Problem and Analysis}

2.1. Assumptions. The conducting polymer film will adopt a hemispherical geometry upon electrodepositing them on to a microelectrode support (Figure 1). If such a geometry is assumed then the substrate will exhibit spherical diffusion both in solution immediately adjacent to the polymer film and within the polymer film itself. We assume that the substrate exhibits Michaelis-Menten kinetics when it reacts at a site within the polymer film. The substrate exhibits firstorder kinetics approximation when the substrate concentration is low. For higher values of the substrate concentration a kinetic rate law based on the Michaelis-Menten equation is more appropriate. We also assume that the partition coefficient for the substrate is unity.

2.2. Neglecting Substrate Concentration Polarization in Solution. Initially, the substrate diffusion in solution, the transport, and kinetic processes within the polymer film were all neglected. On the other hand, the reaction/diffusion equation under steady-state condition corresponding to the normalized substrate concentration within the polymer film can be expressed as [24]

$$
\frac{d^{2} u}{d \rho^{2}}+\frac{2}{\rho} \frac{d u}{d \rho}-\frac{\gamma u}{1+\alpha u}=0 .
$$




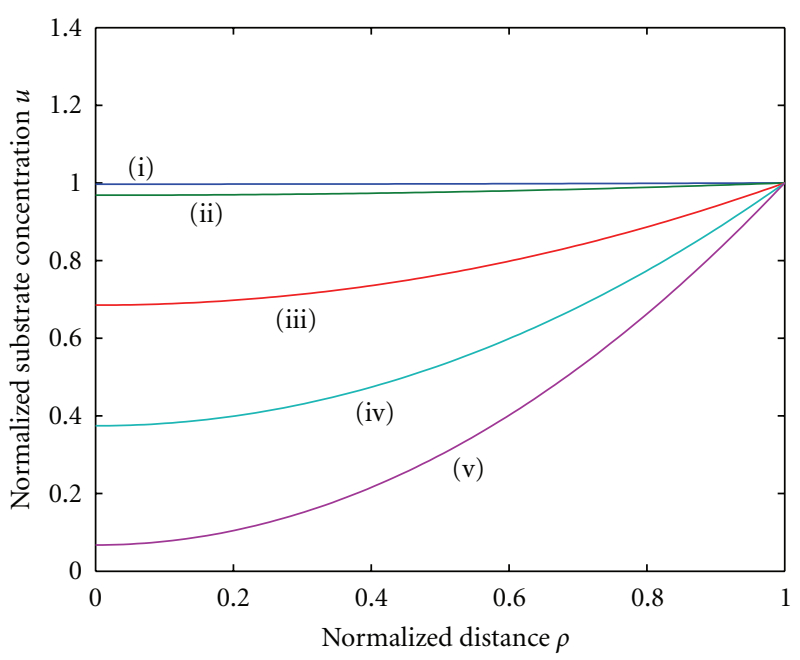

(a)

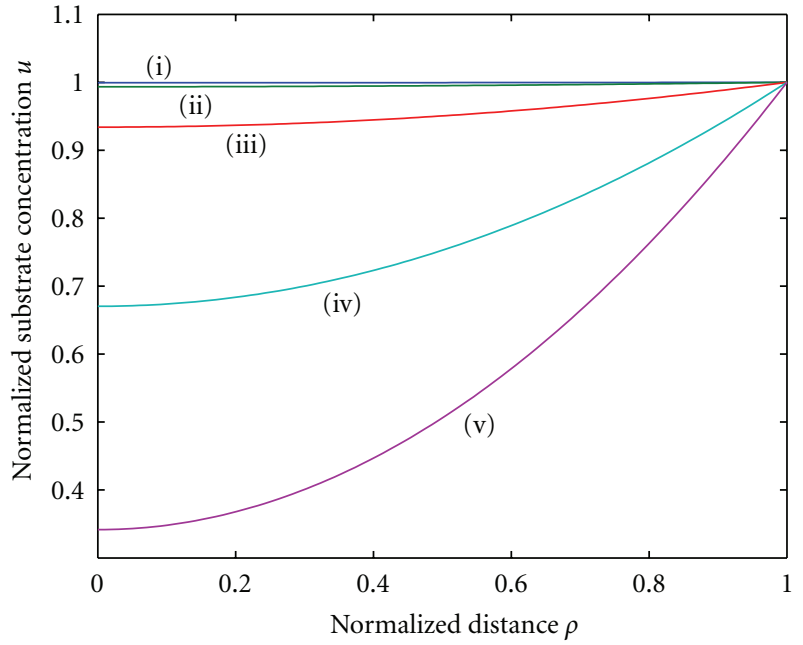

(b)

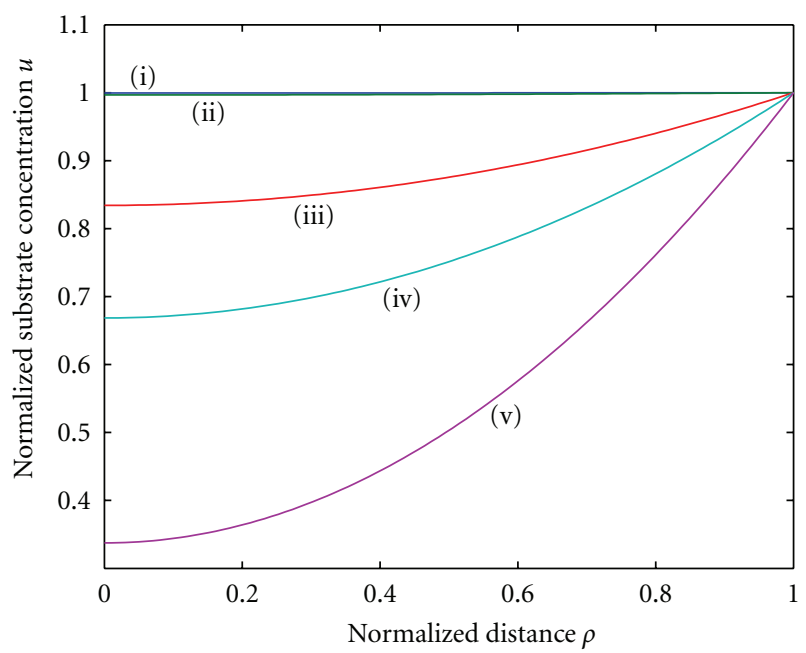

(c)

FIGURE 3: Plot of normalized substrate concentration " $u$ " at a microelectrode modified with a polymer film as a function of " $\rho$ ". The concentrations were computed using (8) for various values of the reaction diffusion parameter " $\gamma$ " when (a) $\alpha=10$ for (i) $\gamma=0.1$, (ii) $\gamma=1$, (iii) $\gamma=10$, (iv) $\gamma=20$, and (v) $\gamma=30$, (b) $\alpha=50$ for (i) $\gamma=0.1$, (ii) $\gamma=1$, (iii) $\gamma=10$, (iv) $\gamma=50$, and (v) $\gamma=100$, and (c) when $\alpha=100$ for (i) $\gamma=0.1$, (ii) $\gamma=1$, (iii) $\gamma=50$, (iv) $\gamma=100$, and (v) $\gamma=200$.

In (1), normalized substrate concentration $u=s / s^{\infty}$, where $s$ denotes the substrate concentration within the polymer film and $s^{\infty}$ denotes the bulk concentration of substrate. The saturation parameter $\alpha=s^{\infty} / K_{M}$, where $K_{M}$ denotes the Michaelis constant. The reaction/diffusion parameter $\gamma$ is given by $\gamma=k_{c} c_{\Sigma} a^{2} / K_{M} D_{S}$, where $k_{c}$ represents the reaction rate constant, $a$ denotes the radius of the microelectrode. $c_{\Sigma}$ denotes the total catalyst concentration in the film, and $D_{S}$ is the diffusion coefficient of the substrate within the polymer film. The normalized distance parameter $\rho$ is given by $r / a$ (where $r$ represents the radial variable). The boundary conditions pertaining to the normalized form are

$$
\begin{array}{cl}
\rho=0, & \frac{d u}{d \rho}=0, \\
\rho=1, & u=1 .
\end{array}
$$

The boundary condition (2a) states that the substrate is electroinactive at the disk. The normalized current density is defined as

$$
\psi=\frac{i a}{n F A D_{S} \mathcal{S}^{\infty}}=\left(\frac{d u}{d \rho}\right)_{\rho=1} .
$$

2.3. Unsaturated (First-Order) Catalytic Kinetics. We initially consider the situation where the substrate concentration in the film is less than the Michaelis constant $K_{M}$. This situation will pertain when the product $\alpha u \ll 1$. Hence (1) reduces to

$$
\frac{d^{2} u}{d \rho^{2}}+\frac{2}{\rho} \frac{d u}{d \rho}-\gamma u=0
$$




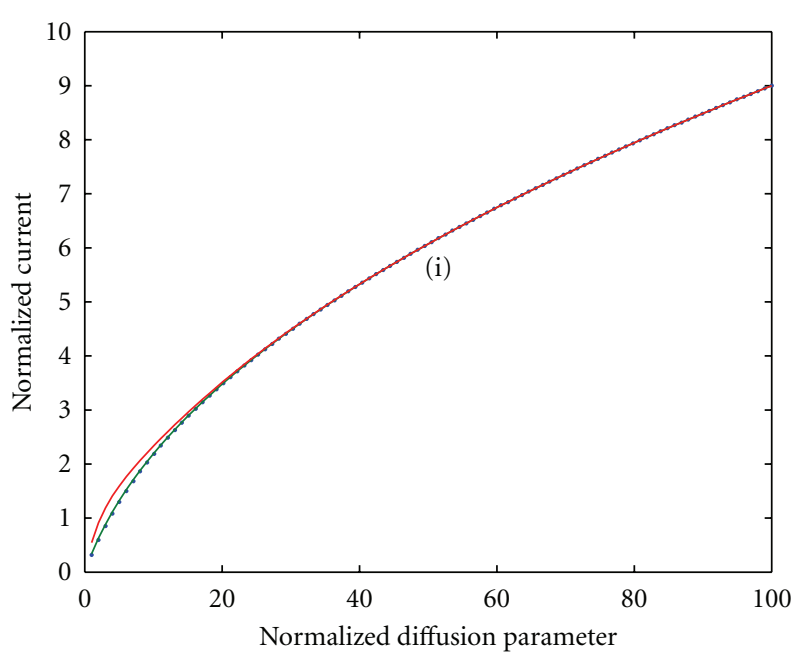

(a)

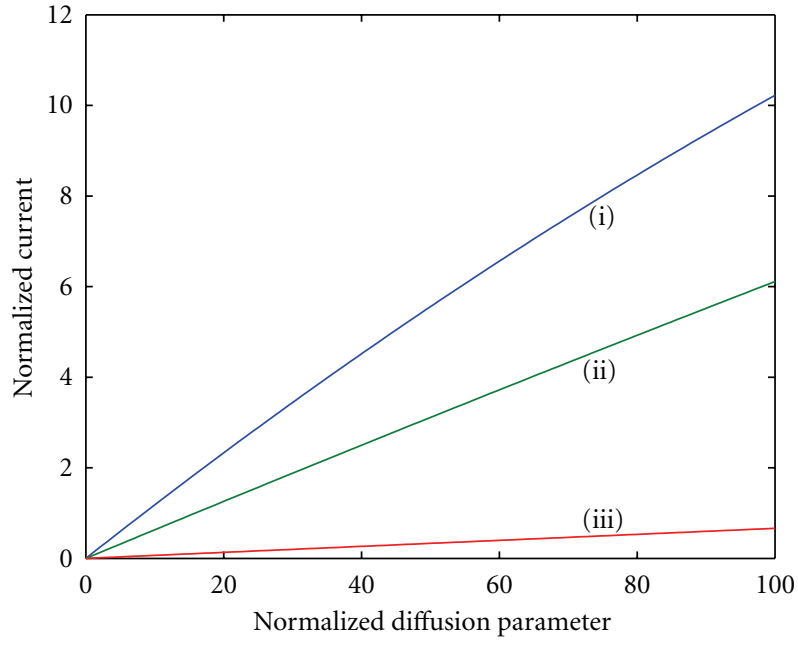

(b)

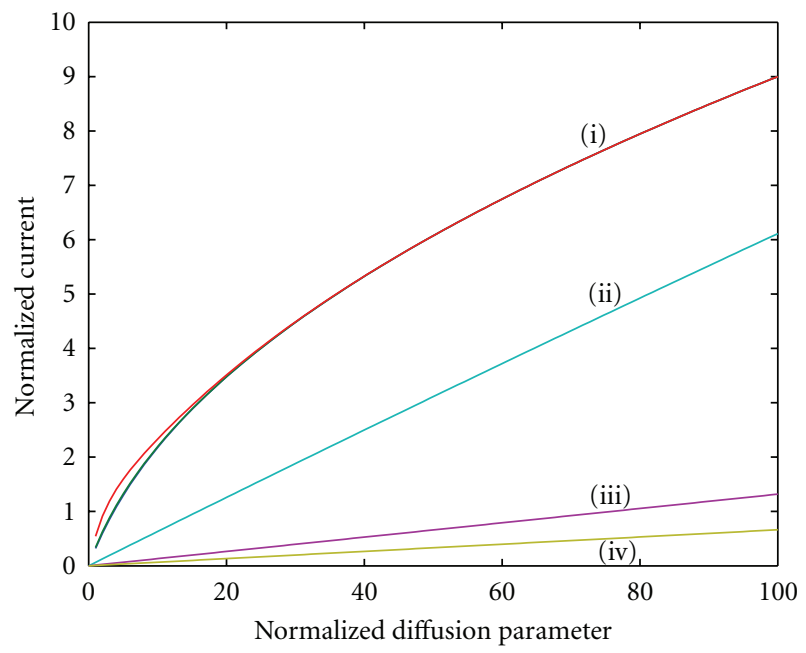

(c)

FIGURE 4: Plot of normalized current " $\psi$ " versus the reaction/diffusion parameter " $\gamma$ " at a microelectrode modified using a conducting polymer film. (a) (i) $\alpha=0.1,1$, the current was calculated using (6) and (11) for all small and medium values of $\alpha$. (b) (i) $\alpha=5$, (ii) $\alpha=10$, (iii) $\alpha=100$, the current was calculated using (9) for all large values of “ $\alpha$ ". (c) (i) $\alpha=0.01,0.1,1$ (ii) $\alpha=10$, (iii) $\alpha=50$, and, (iv) $\alpha=100$, the current was calculated using (9) and (11) (solid line) (11); (dotted line) (6). Solid lines are compared with points.

By solving (4), we can obtain the expression for the normalized substrate concentration as follows:

$$
u(\rho, \gamma)=\frac{\sinh (\sqrt{\gamma} \rho)}{\rho \sinh (\sqrt{\gamma})} .
$$

Also the expression of the normalized steady-state current density is given below

$$
\psi(\gamma)=\sqrt{\gamma} \operatorname{coth}(\sqrt{\gamma})-1
$$

\subsection{For Large Values of the Saturation Parameter $\alpha$ and All} Values of the Reaction/Diffusion Parameter $\gamma$. We now consider the limiting situation where the substrate concentration in the film is very much greater than the Michaelis constant $K_{M}$. In this case $\alpha u \gg 1$ and (1) reduces to

$$
\frac{d^{2} u}{d \rho^{2}}+\frac{2}{\rho} \frac{d u}{d \rho}-\frac{\gamma}{\alpha}+\frac{\gamma}{\alpha^{2} u}=0 .
$$

We obtain the approximate expression of normalized concentration of substrate as

$u(\rho, \gamma, \alpha)=1+\frac{\gamma\left(12 \alpha^{2}-6 \alpha-\gamma\right)}{36 \alpha^{3}}\left(\rho^{2}-1\right)+\frac{\gamma^{2}}{120 \alpha^{3}}\left(\rho^{4}-1\right)$.

Using (3), we obtain the expression of the normalized current density as

$$
\psi(\gamma, \alpha)=\frac{\gamma\left(30 \alpha^{2}-15 \alpha-\gamma\right)}{45 \alpha^{3}} .
$$




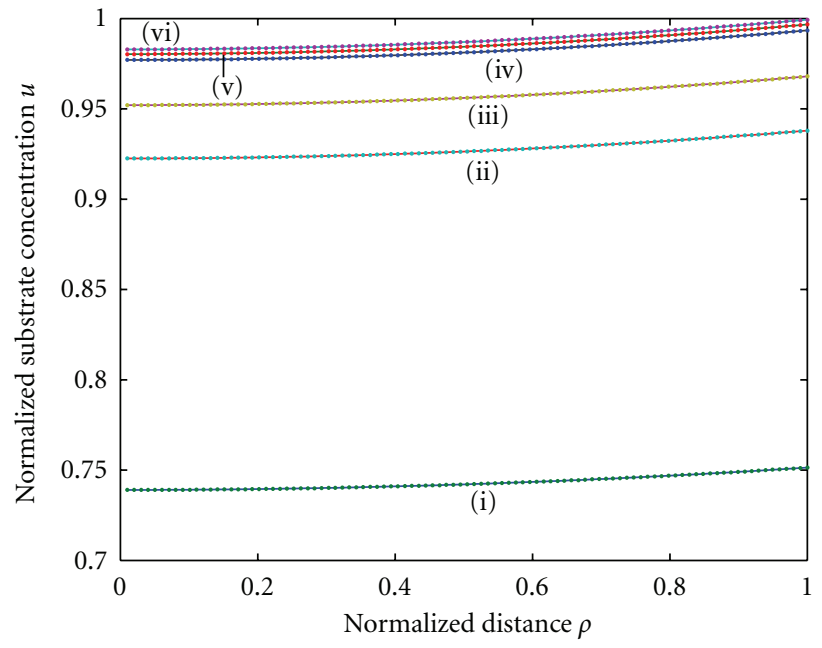

(a)

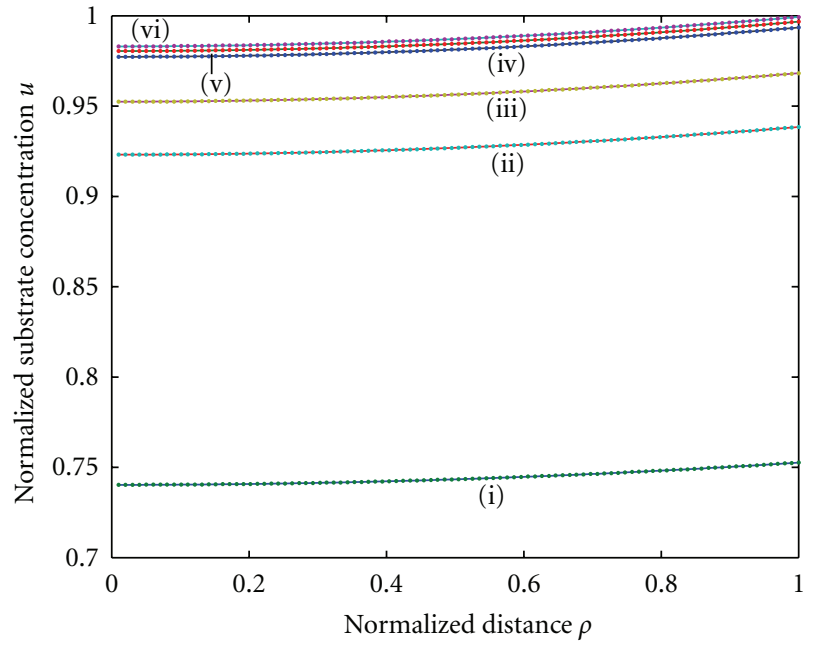

(b)

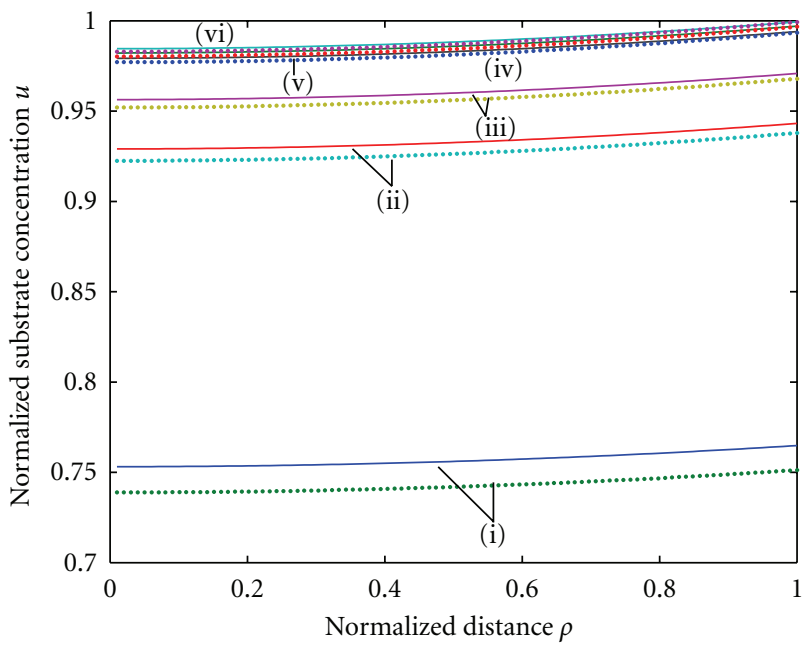

(c)

FIGURE 5: Plot of normalized substrate concentration " $u$ " at a microelectrode modified with a conducting polymer film as a function of " $\rho$ ". The concentrations were computed for various values of Biot number " $v$ " when (a) $\alpha=0.001, \gamma=0.1$, (b) $\alpha=0.01, \gamma=0.1$, and (c) $\alpha=0.1, \gamma=0.1$. (solid line) (22); (dotted line) (17). Solid lines are compared with points. In this figure (i) $v=0.1$, (ii) $v=0.5$, (iii) $v=1$, (iv) $v=5$, (v) $v=10$, (vi) $v=50$.

The above approximation will be valid for all values of diffusion parameter $\gamma$ and large values of the saturation parameter $\alpha$.

\subsection{For Small and Medium Values of the Saturation Parameter} $\alpha$ and All Values of the Reaction/Diffusion Parameter $\gamma$. In recent days, homotopy perturbation method is often employed to solve several analytical problems. In addition, several groups demonstrated the efficiency and suitability of the HPM for solving nonlinear equations and other electrochemical problems [26-29]. He [30] used HPM to solve the Lighthill equation, the Duffing equation [31], and the Blasius equation [32]. This method has also been used to solve nonlinear boundary value problems [33], integral equation [34-36], Klein-Gordon and Sine-Gordon equations [37], Emden-Flower-type equations [38], and several other problems [39-41]. Using homotopy perturbation method (refer to Appendix A), the approximate solution of (1) is

$$
u(\rho, \gamma, \alpha)=\frac{\sinh (\sqrt{\gamma} \rho)}{\rho \sinh (\sqrt{\gamma})}-\frac{\alpha \gamma \sinh (\sqrt{\gamma} \rho)}{\rho \sinh ^{3}(\sqrt{\gamma})}+\frac{\alpha \gamma}{\sinh ^{2}(\sqrt{\gamma})} .
$$

The normalized concentration of the substrate satisfies the boundary conditions (2a) and (2b). The expression of the normalized current density becomes

$$
\begin{aligned}
\psi(\gamma, \alpha)= & \sqrt{\gamma} \operatorname{coth}(\sqrt{\gamma})-1 \\
& +\alpha \gamma \operatorname{cosech}^{2}(\sqrt{\gamma})\lfloor\sqrt{\gamma} \operatorname{coth}(\sqrt{\gamma})-1\rfloor .
\end{aligned}
$$

Equations (10) and (11) represent approximate expressions of normalized substrate concentration and current density 


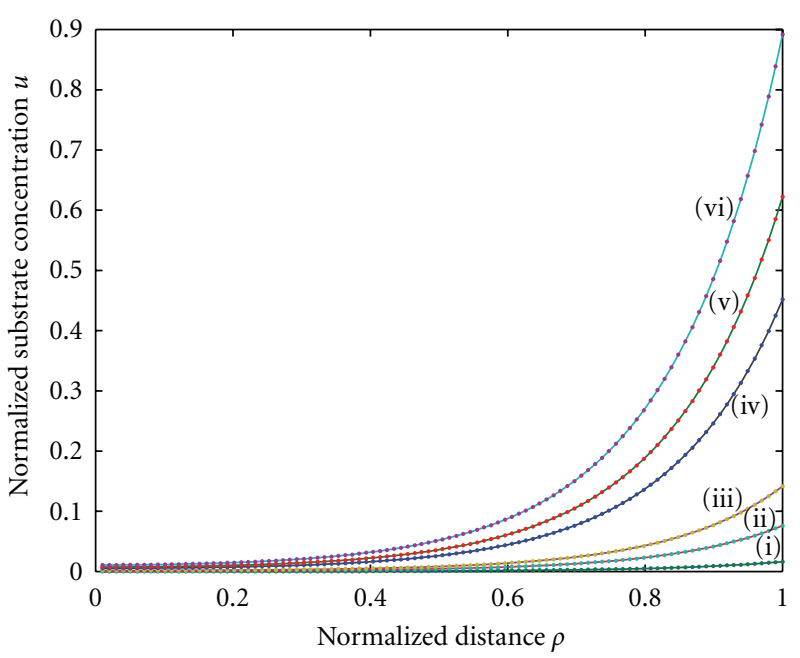

(a)

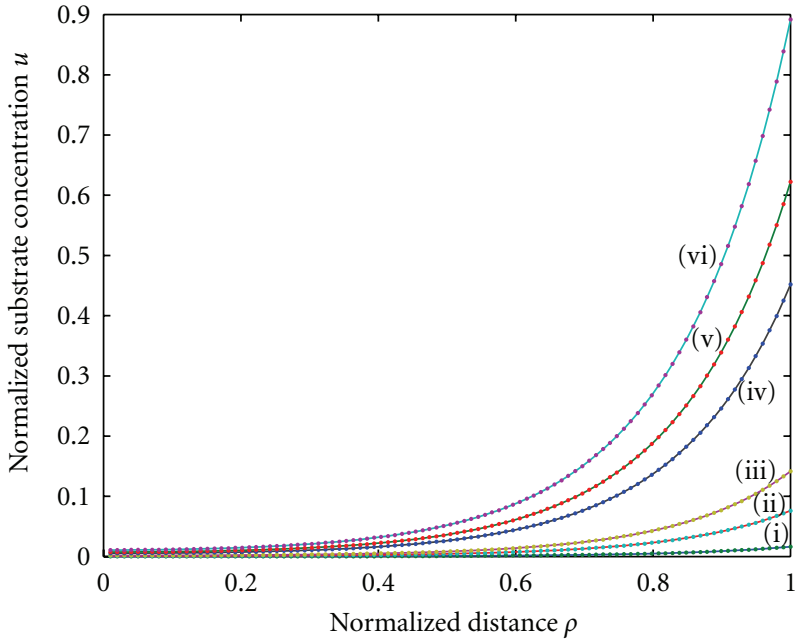

(b)

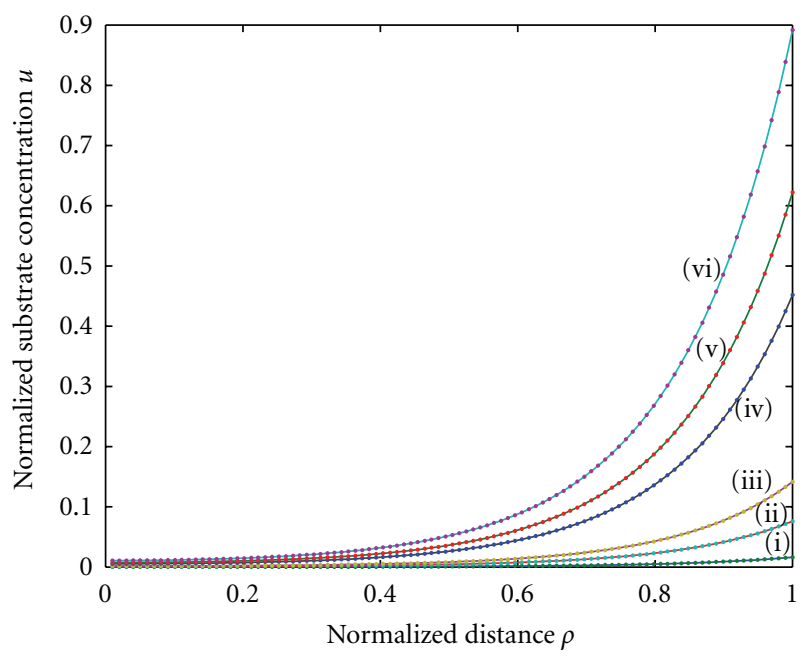

(c)

FIGURE 6: Normalized substrate concentration " $u$ " at a microelectrode modified with a conducting polymer film as a function of " $\rho$ ". The concentrations were computed for various values of the Biot number " $v$ " when (a) $\alpha=0.001, \gamma=50,(\mathrm{~b}) \alpha=0.01, \gamma=50$, and (c) $\alpha=0.1, \gamma=50$. (solid line) (22); (dotted line) (17). Solid lines are compared with points. In this figure (i) $v=0.1$, (ii) $v=0.5$, (iii) $v=1$, (iv) $v=5$, (v) $v=10$, (vi) $v=50$.

for all small and medium values of the saturation parameter $\alpha$ and all values of the diffusion parameter $\gamma$.

2.6. Discussion. The kinetic response of a microelectrode depends on the concentration of substrate. The concentration of substrate depends on the following two factors $\gamma$ and $\alpha$. The diffusion parameter $\gamma$ represents the ratio of the characteristic time of the enzymatic reaction to that of substrate diffusion. This parameter can be varied by changing either the radius of the microelectrode or the amount of catalyst in conducting polymer ultramicroelectrodes. This parameter describes the relative importance of diffusion and reaction in conducting polymer ultramicroelectrodes. When $\gamma$ is small, the kinetics are dominant resistance; the uptake of substrate in the polymer film is kinetically controlled. Under these conditions, the substrate concentration profile across the microelectrode is essentially uniform. The overall kinetics are determined by the total amount of active catalyst $c_{\Sigma}$. When the diffusion parameter $\gamma$ is large, diffusion limitations are the principal determining factor. In both the unsaturated and saturated situations (small and large values of $\alpha$ ), the current response increases as $\gamma$ increases. This is to be expected as the reaction kinetics become more facile.

The approximate expressions of concentration of substrate and current density for various values of $\alpha$ and $\gamma$ are reported in Table 1. In the case when the substrate diffusion in the adjacent solution is neglected, the expression corresponding to the concentration of substrate (5) and current (6) was provided by Lyons et al. [24] (refer to Table 1).

Figure 2 represents the substrate concentration $u$ for various values of the reaction diffusion parameter $y$ and for $\alpha \leq 1$. From Figure 2, it is evident that the normalized 


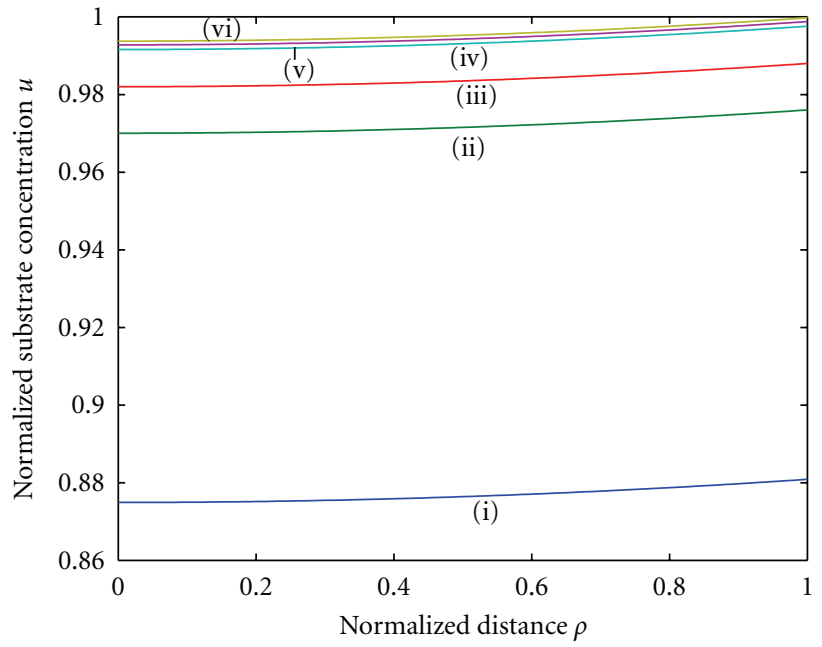

(a)

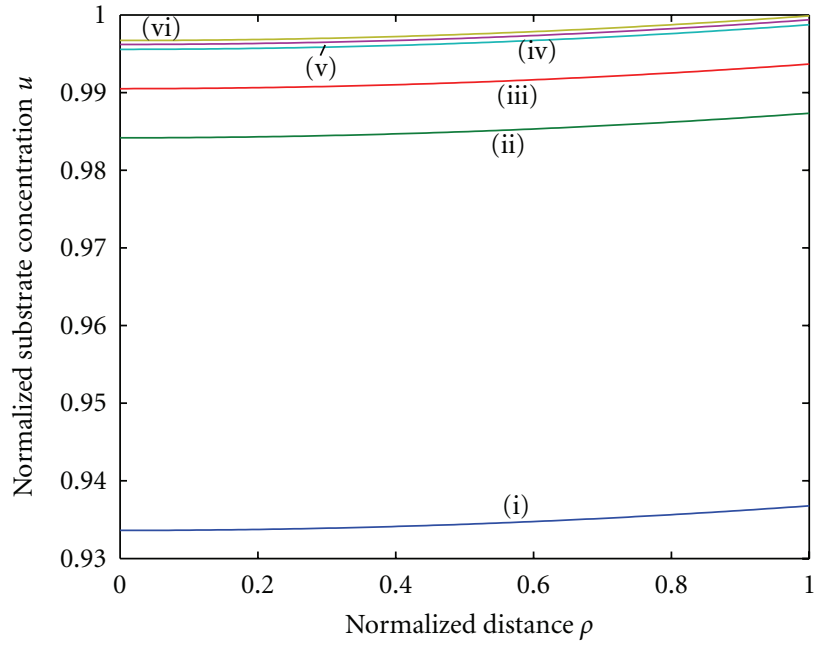

(b)

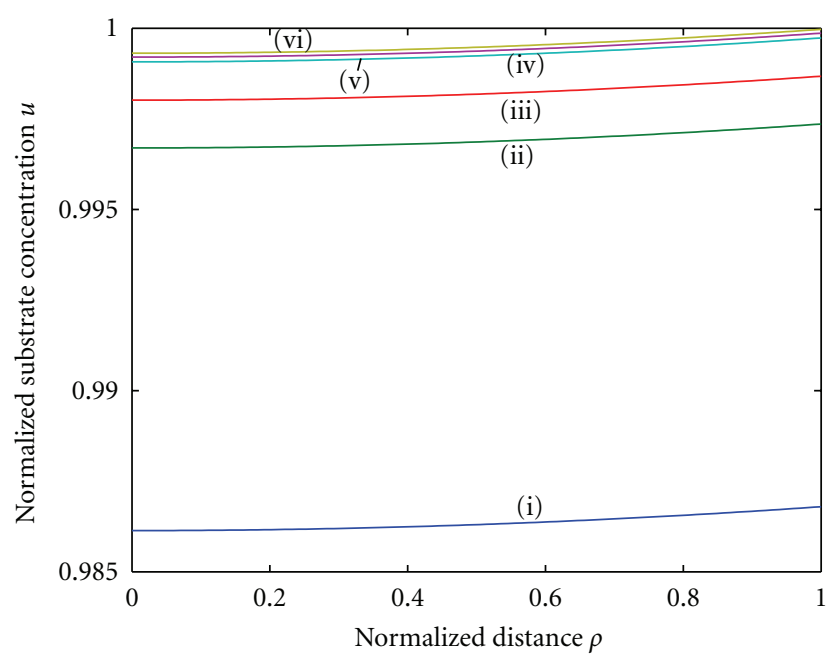

(c)

FIGURE 7: Plot of Normalized substrate concentration " $u$ " at a microelectrode modified with a polymer film as a function of " $\rho$ ". The concentrations were computed using (20) for various values of the Biot number " $v$ " when $\gamma=0.1$ and for (a) $\alpha=5$, (b) $\alpha=10$ and (c) $\alpha=50$. In this figure (i) $v=0.1$, (ii) $v=0.5$, (iii) $v=1$, (iv) $v=5$, (v) $v=10$, (vi) $v=50$.

steady-state substrate concentration $u$ reaches the maximum value 1 , when $\rho=1$. Figure 3 indicates the values of substrate concentrations for large values of $\alpha(\alpha \geq 10)$ and all values of $\gamma$. From Figure 3, the value of concentration $u$ is inversely proportional to the value of the reaction diffusion parameter $\gamma$. When $\gamma$ is small $(\gamma \leq 1)$, the substrate concentration profile across the microelectrode is uniform (refer to Figures 2 and 3$)$.

Figure 4 indicates the normalized steady-state current $\psi$ for all values of $\alpha$. From Figure 4(a), it is noticed that our analytical results (9) and (11) agree with the limiting result of Lyons et al. [24] work. The normalized steady-state current for all large values of $\alpha$ is calculated using (9) in Figure 4(b). A series of normalized current density for all values of $\alpha$ is plotted in Figure 4(c). From Figures 4(b) and 4(c), it is evident that the value of the current decreases when $\alpha$ increases as $\gamma$ or radius of the electrode increases.

\section{Problem Resolution including Substrate Concentration Polarization in Solution}

Here, we include the substrate diffusion in the solution adjacent to the polymer film. In this case transport and kinetics are described by (1), but the boundary conditions are given by [24]

$$
\begin{gathered}
\frac{d u}{d \rho}=0 \quad \text { at } \rho=0, \\
u=u_{1} \quad \text { at } \rho=1, \\
\frac{d u}{d \rho}=v\left(1-u_{1}\right) \quad \text { at } \rho=1,
\end{gathered}
$$




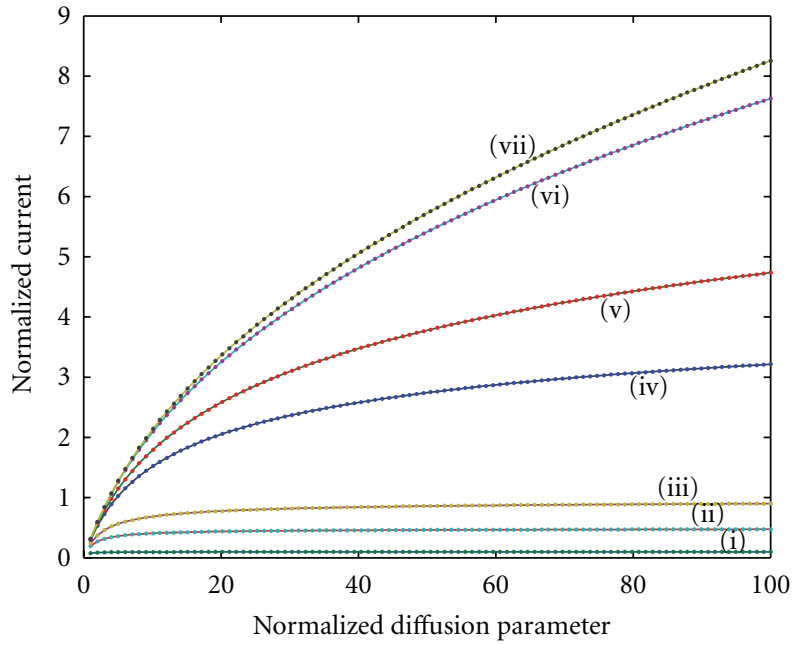

(a)

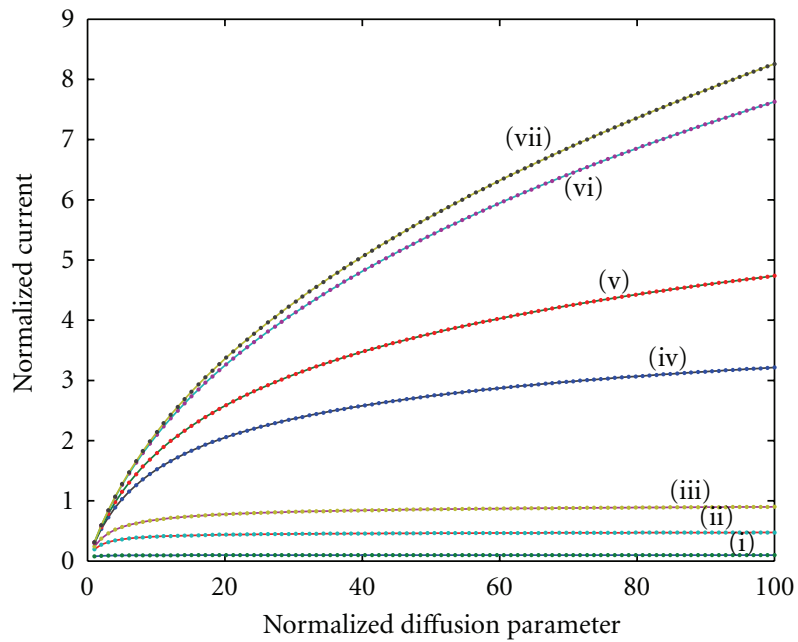

(b)

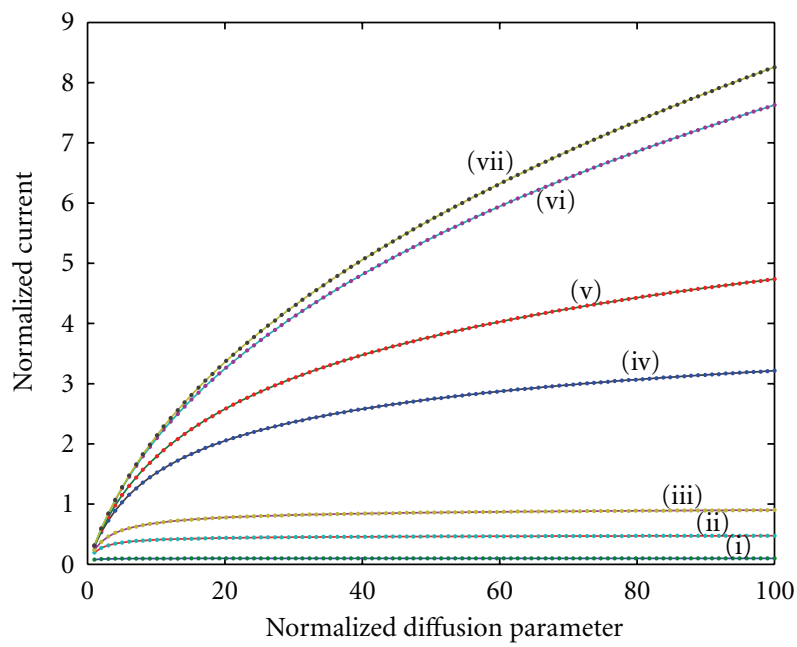

(c)

FIGURE 8: Normalized current " $\psi$ " versus the reaction/diffusion parameter " $\gamma$ " at a microelectrode modified using a conducting polymer film. The current was calculated for various values of the Biot number " $v$ " when (a) $\alpha=0.001$, (b) $\alpha=0.01$, and (c) $\alpha=0.1$. (solid line) (23); (dotted line) (18). Solid lines are compared with points. In this figure (i) $v=0.1$, (ii) $v=0.5$, (iii) $v=1$, (iv) $v=5$, (v) $v=10$, (vi) $v=50$, and (vii) $v=100$.

where the Biot number $v$ has been introduced

$$
v=\frac{k_{D}^{\prime}}{k_{D}},
$$

where $k_{D}^{\prime}$ represents the diffusional rate constant of the substrate in solution and $k_{D}$ is the value that represents the transport of substrate within polymer film. The diffusional rate constant $k_{D}$ to a microelectrode is given by

$$
k_{D}=\frac{4 D_{S}}{\pi a} .
$$

Using (13), we obtain

$$
v=\frac{4}{\pi}\left(\frac{D_{S}^{\prime}}{D_{S}}\right) .
$$

3.1. Unsaturated (First-Order) Catalytic Kinetics. Initially, we considered a situation where the substrate concentration in the film is less than the Michaelis constant $K_{M}$. This situation will pertain when the product $\alpha u \ll 1$. Hence (1) reduces to

$$
\frac{d^{2} u}{d \rho^{2}}+\frac{2}{\rho} \frac{d u}{d \rho}-\gamma u=0
$$

By solving (16) using the boundary condition (12a)(12c), we can obtain the analytical expression of normalized concentration of substrate as follows:

$$
u(\rho, \gamma, v)=\frac{\sinh (\sqrt{\gamma} \rho)}{\rho\left\{\sinh (\sqrt{\gamma})+\frac{1}{v}[\sqrt{\gamma} \cosh (\sqrt{\gamma})-\sinh (\sqrt{\gamma})]\right\}} .
$$




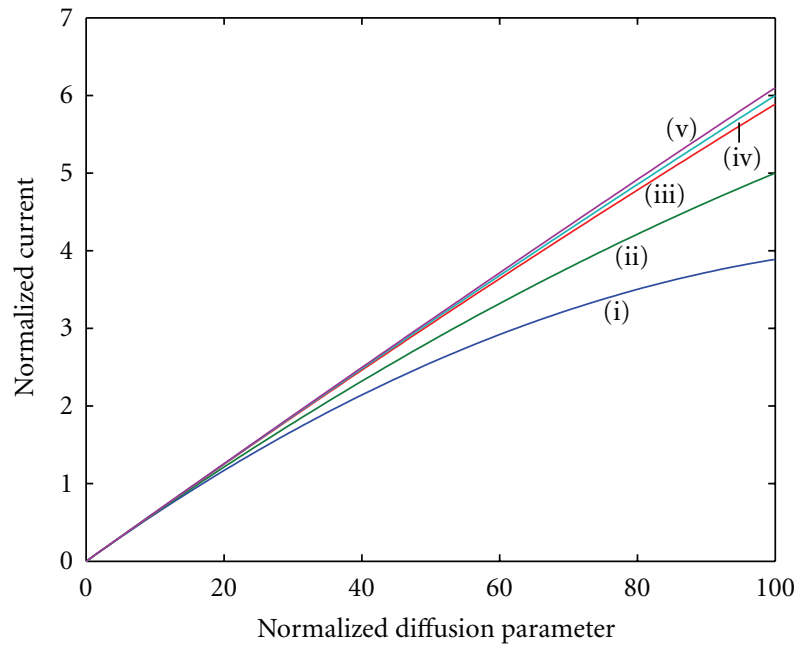

FIGURE 9: Normalized current " $\psi$ " versus the reaction/diffusion parameter " $y$ " at a microelectrode modified using a conducting polymer film. The current was calculated using (21) for various values of the Biot number " $v$ " when $\alpha=10$. In this figure (i) $v=0.5$, (ii) $v=1$, (iii) $v=5$, (iv) $v=10$, and (v) $v=100$.

Also the expression of the normalized current density is shown below

$$
\psi(\gamma, v)=\frac{\sqrt{\gamma} \cosh \lfloor\sqrt{\gamma}\rfloor-\sinh \lfloor\sqrt{\gamma}\rfloor}{\sinh (\sqrt{\gamma})+\frac{1}{v}[\sqrt{\gamma} \cosh (\sqrt{\gamma})-\sinh (\sqrt{\gamma})]} .
$$

The above analytical expression of substrate concentration and current is identical to Lyons et al. [24] work.

3.2. For Large Values of the Saturation Parameter $\alpha$ and All Values of the Reaction/Diffusion Parameter $\gamma$. We now consider the limiting situation where the substrate concentration in the film is very much greater than the Michaelis constant $K_{M}$. In this case $\alpha u \gg 1$ and (1) reduces to

$$
\frac{d^{2} u}{d \rho^{2}}+\frac{2}{\rho} \frac{d u}{d \rho}-\frac{\gamma}{\alpha}+\frac{\gamma}{\alpha^{2} u}=0
$$

By solving (19), we obtain the approximate expression of the normalized concentration of substrate as

$$
\begin{aligned}
u(\rho, \gamma, \alpha, v)= & 1+\frac{\gamma\left(12 \alpha^{2} v-6 \alpha v-\gamma v-2 \gamma\right)}{36 \alpha^{3} v}\left(\rho^{2}-1-\frac{2}{v}\right) \\
& +\frac{\gamma^{2}}{120 \alpha^{3}}\left(\rho^{4}-1-\frac{4}{v}\right) .
\end{aligned}
$$

Also we can obtain the expression of the normalized current density as

$$
\psi(\gamma, \alpha, v)=\frac{\gamma\left(30 \alpha^{2} v-15 \alpha v-\gamma v-5 \gamma\right)}{45 \alpha^{3} v} .
$$

The above approximation will be valid for all values of diffusion parameter $\gamma$ and large values of saturation parameter $\alpha$.

\subsection{For Small and Medium Values of the Saturation Parameter} $\alpha$ and All Values of the Reaction/Diffusion Parameter $\gamma$. Using this homotopy perturbation method, we can obtain the solution of (1)

$$
\begin{aligned}
u(\rho, \gamma, \alpha, v) & \frac{\sinh (\sqrt{\gamma} \rho)}{\rho\left\{\sinh (\sqrt{\gamma})+\frac{1}{v}[\sqrt{\gamma} \cosh (\sqrt{\gamma})-\sinh (\sqrt{\gamma})]\right\}} \\
& -\frac{\alpha \gamma \sinh (\sqrt{\gamma} \rho)}{\rho\left\{\sinh (\sqrt{\gamma})+\frac{1}{v}[\sqrt{\gamma} \cosh (\sqrt{\gamma})-\sinh (\sqrt{\gamma})]\right\}^{3}} \\
& +\frac{\alpha \gamma}{\left\{\sinh (\sqrt{\gamma})+\frac{1}{v}[\sqrt{\gamma} \cosh (\sqrt{\gamma})-\sinh (\sqrt{\gamma})]\right\}^{2}} .
\end{aligned}
$$

The above equation satisfies the boundary conditions (12a)(12c). The expression of the normalized current density becomes

$$
\begin{aligned}
\psi(\gamma, \alpha, v)= & \frac{\sqrt{\gamma} \cosh \lfloor\sqrt{\gamma}\rfloor-\sinh \lfloor\sqrt{\gamma}\rfloor}{\sinh (\sqrt{\gamma})+\frac{1}{v}[\sqrt{\gamma} \cosh (\sqrt{\gamma})-\sinh (\sqrt{\gamma})]} \\
& -\frac{\alpha \gamma(\sqrt{\gamma} \cosh \lfloor\sqrt{\gamma}\rfloor-\sinh \lfloor\sqrt{\gamma}\rfloor)}{\left\{\sinh (\sqrt{\gamma})+\frac{1}{v}[\sqrt{\gamma} \cosh (\sqrt{\gamma})-\sinh (\sqrt{\gamma})]\right\}^{3}} .
\end{aligned}
$$

Equations (22) and (23) represent a new closed form of approximate expressions of normalized substrate concentration and current density for small and medium of parameters $\alpha$ and all values of $\gamma$.

3.4. Discussion. In the case when the substrate concentration is very low, the expression corresponding to the concentration of substrate (17) and current (18) was provided by Lyons et al. [24] (refer to Table 2). Figure 5 represents the normalized steady-state substrate concentration $u$ at a polymercoated microelectrode. The concentration of substrate was calculated for all small values of the saturation parameter $\alpha$. From Figure 5, it is inferred that the concentration increases when $v$ increases. Also for any fixed values of $v$ and small values of $\alpha$ and $\gamma$, the concentration is uniform throughout the film.

The normalized steady-state substrate concentration $u$ is plotted for all small values of the saturation parameter $\alpha$ in Figure 6. From Figure 6, it is evident that when the values of the Biot number $v$ increase, the values corresponding to the substrate concentration $u$ also increase when $\gamma \geq$ 50. Our analytical results agree with the limiting result of 
Lyons et al. [24] work. Figure 7 indicates the values of substrate concentrations for large values of $\alpha(\alpha \geq 5)$ and all values of the Biot number $v$. From Figure 7 , it is inferred that $u \approx 1$ when $v \geq 50$ and $\gamma \leq 0.1$.

Figure 8 represents the normalized current density $\psi$ for all values of the Biot number $v$. In addition, from Figure 8, we noticed that the normalized current density increases as the Biot number $v$ increases. Normalized current density $\psi$ versus $\gamma$ for various values of the Biot number $v$ and for large values of $\alpha$ is plotted using (21) in Figure 9. From Figure 9, it is evident that the value of the current increases when the Biot number $v$ increases.

\section{Conclusions}

The steady-state amperometric response for a conducting polymer microelectrode system which exhibits MichaelisMenten kinetics has been discussed. We have presented a mathematical model of reaction and diffusion within a conducting polymer film which is deposited on a support surface of micrometer dimensions. Approximate analytical solutions of the nonlinear reaction diffusion equation have been derived. Analytical expressions of substrate concentration within the polymer film are derived for all values of the diffusion parameter and the saturation parameter using homotopy perturbation method. The analytical results derived therein may be used to predict the steady-state sensor response on experimental values, and the theoretical value of surface concentration for which the amperometric response is nonlinear.

\section{Appendix}

\section{Solution of (1) Using Homotopy Perturbation Method}

In this appendix, we indicate how (10) in this paper is derived. To find the solution of (1), we first construct a homotopy as follows:

$$
\begin{aligned}
& (1-p)\left[\frac{d^{2} u}{d \rho^{2}}+\frac{2}{\rho} \frac{d u}{d \rho}-\gamma u\right] \\
& +p\left[(1+\alpha u)\left(\frac{d^{2} u}{d \rho^{2}}+\frac{2}{\rho} \frac{d u}{d \rho}\right)-\gamma u\right]=0 .
\end{aligned}
$$

The approximate solution of (A.1) is given by

$$
u=u_{0}+p u_{1}+p^{2} u_{2}+p^{3} u_{3}+\cdots .
$$

Substituting (A.2) into (A.1) and comparing the coefficients of like powers of $p$, we get

$$
\begin{gathered}
p^{0}: \frac{d^{2} u_{0}}{d \rho^{2}}+\frac{2}{\rho} \frac{d u_{0}}{d \rho}-\gamma u_{0}=0 \\
p^{1}: \frac{d^{2} u_{1}}{d \rho^{2}}+\frac{2}{\rho} \frac{d u_{1}}{d \rho}-\gamma u_{1}+\alpha u_{0} \frac{d^{2} u_{0}}{d \rho^{2}}+\frac{2}{\rho} \alpha u_{0} \frac{d u_{0}}{d \rho}=0 .
\end{gathered}
$$

The initial approximations are as follows:

$$
\begin{gathered}
u_{0}(\rho=1)=1, \quad\left(\frac{d u_{0}}{d \rho}\right)_{\rho=0}=0, \\
u_{i}(\rho=1)=0, \quad\left(\frac{d u_{i}}{d \rho}\right)_{\rho=0}=0, \quad \forall i=1,2,3, \ldots
\end{gathered}
$$

Upon solving (A.3) and (A.4) and using the boundary conditions (A.5) and (A.6), we get

$$
\begin{gathered}
u_{0}(\rho)=\frac{\sinh (\sqrt{\gamma} \rho)}{\rho \sinh (\sqrt{\gamma})}, \\
u_{1}(\rho)=-\frac{\alpha \gamma \sinh (\sqrt{\gamma} \rho)}{\rho \sinh ^{3}(\sqrt{\gamma})}+\frac{\alpha \gamma}{\sinh ^{2}(\sqrt{\gamma})} .
\end{gathered}
$$

$u_{1}(\rho)$ is valid only when $\alpha$ and $\gamma$ are small. According to the HPM, we can conclude that

$$
u(\rho)=\lim _{p \rightarrow 1} u(\rho) \approx u_{0}+u_{1}
$$

Using (A.7) and (A.8) in (A.9), we obtain the final result as described in (10). Similarly (22) can also be obtained.

\section{Acknowledgments}

This work is supported by the Council of Scientific and Industrial Research (CSIR), Government of India. The authors are thankful to the Secretary, the Principal, The Madura College, Madurai, India for their constant encouragement. They thank the reviewers for their valuable comments to improve the quality of the paper.

\section{References}

[1] W. E. Morf, "Theoretical treatment of the amperometric current response of multiple microelectrode arrays," Analytica Chimica Acta, vol. 330, no. 2-3, pp. 139-149, 1996.

[2] M. I. Montenegro, M. A. Queiros, and J. L. Daschbach, Microelectrodes: Theory and Applications, Kluwer, Dordrecht, The Netherlands, 1991.

[3] R. M. Wightman, "Microvoltammetric electrodes," Analytical Chemistry, vol. 53, pp. 1125A-1134A, 1981.

[4] K. R. Wehmeyer, M. R. Deakin, and R. M. Wightman, "Electroanalytical properties of band electrodes of submicrometer width," Analytical Chemistry, vol. 57, no. 9, pp. 1913-1916, 1985.

[5] R. M. Wightman, P. Runnels, and K. Troyer, "Analysis of chemical dynamics in microenvironments," Analytica Chimica Acta, vol. 400, no. 1-3, pp. 5-12, 1999.

[6] J. Park, V. Quaiserová-Mocko, K. Pecková, J. J. Galligan, G. D. Fink, and G. M. Swain, "Fabrication, characterization, and application of a diamond microelectrode for electrochemical measurement of norepinephrine release from the sympathetic nervous system," Diamond and Related Materials, vol. 15, no. 4-8, pp. 761-772, 2006. 
[7] S. Alwarappan, K. S. A. Butcher, and D. K. Y. Wong, "Evaluation of hydrogenated physically small carbon electrodes in resisting fouling during voltammetric detection of dopamine," Sensors and Actuators B, vol. 128, no. 1, pp. 299-305, 2007.

[8] Y. Takahashi, T. Miyamoto, S. Hitoshi et al., "Electrochemical detection of epidermal growth factor receptors on a single living cell surface by scanning electrochemical microscopy," Analytical Chemistry, vol. 81, no. 7, pp. 2785-2790, 2009.

[9] M. V. Mirkin, F. R. F. Fan, and A. J. Bard, "Scanning electrochemical microscopy part 13. Evaluation of the tip shapes of nanometer size microelectrodes," Journal of Electroanalytical Chemistry, vol. 328, no. 1-2, pp. 47-62, 1992.

[10] B. Liu, S. A. Rotenberg, and M. V. Mirkin, "Scanning electrochemical microscopy of living cells: different redox activities of nonmetastatic and metastatic human breast cells," Proceedings of the National Academy of Sciences of the United States of America, vol. 97, no. 18, pp. 9855-9860, 2000.

[11] P. Sun, F. O. Laforge, T. P. Abeyweera, S. A. Rotenberg, J. Carpino, and M. V. Mirkin, "Nanoelectrochemistry of mammalian cells," Proceedings of the National Academy of Sciences of the United States of America, vol. 105, no. 2, pp. 443448, 2008.

[12] J. Mauzeroll, A. J. Bard, O. Owhadian, and T. J. Monks, "Menadione metabolism to thiodione in hepatoblastoma by scanning electrochemical microscopy," Proceedings of the National Academy of Sciences of the United States of America, vol. 101, no. 51, pp. 17582-17587, 2004.

[13] A. R. Hillman and R. G. Linford, Electrochemical Science and Technology of Polymers, Elsevier, Amsterdam, The Netherlands, 1987.

[14] M. E. G. Lyons, Electroactive Polymer Electrochemistry: Part I, Fundamental, Plenum Press, New York, NY, USA, 1994.

[15] M. E.G. Lyons, "Transport and kinetics in electroactive polymers," Advances in Chemical Physics, vol. 94, pp. 297-624, 1996.

[16] G. P. Evans, H. Gerisher, and C. W. Tobias, Advances in Electrochemical Science and Engineering, VCH, Weinheim, Germany, 1990.

[17] S. A. Wring and J. P. Hart, "Chemically modified, carbonbased electrodes and their application as electrochemical sensors for the analysis of biologically important compounds. A review," Analyst, vol. 117, pp. 1215-1229, 1992.

[18] R. W. Murray, Molecular Design of Electrode Surfaces, Techniques of Chemistry Series, Wiley-Interscience, New York, NY, USA, 1992.

[19] P. G. Edelman and J. Wang, Biosensors and Chemical Sensors, Optimising Performance through Polymeric Materials, ACS Symposium Series, American Chemical Society, Washington, DC, USA, 1992.

[20] M. E. G. Lyons, J. C. Greer, C. A. Fitzgerald, T. Bannon, and P. N. Barlett, "Reaction/diffusion with Michaelis-Menten kinetics in electroactive polymer films part 1 . The steady-state amperometric response," Analyst, vol. 121, no. 6, pp. 715-731, 1996.

[21] M. E.G. Lyons, "Reaction/diffusion with Michaelis-Menten kinetics in electroactive polymer films-part 2: the transient amperometric response," Analyst, vol. 123, no. 10, pp. 19471959, 1998.

[22] S. Dong and G. Che, "Electrocatalysis at a microdisk electrode modified with a redox species," Journal of Electroanalytical Chemistry and Interfacial Electrochemistry, vol. 309, no. 1-2, p. 103, 1991.

[23] G. Che and S. Dong, "Electrochemical catalysis at an ultramicroelectrode modified with redox species," Electrochimica Acta, vol. 38, no. 4, pp. 581-588, 1993.
[24] M. E. G. Lyons, T. Bannon, and S. Rebouillat, "Reaction/diffusion at conducting polymer ultramicroelectrodes," Analyst, vol. 123, no. 10, pp. 1961-1966, 1998.

[25] S. Anitha, A. Subbiah, and L. Rajendran, "Solutions of the coupled reaction and diffusion equations within polymermodified ultramicroelectrodes," The Journal of Physical Chemistry A, vol. 114, no. 26, pp. 7030-7037, 2010.

[26] Q. K. Ghori, M. Ahmed, and A. M. Siddiqui, "Application of homotopy perturbation method to squeezing flow of a newtonian fluid," International Journal of Nonlinear Sciences and Numerical Simulation, vol. 8, no. 2, pp. 179-184, 2007.

[27] T. Ozis and A. Yildirim, "A comparative study of He's Homotopy Perturbation Method for determining frequency-amplitude relation of a nonlinear oscillator with discontinuities," International Journal of Nonlinear Sciences and Numerical Simulation, vol. 8, no. 2, pp. 243-248, 2007.

[28] S.-J. Li and Y.-X. Liu, "An improved approach to nonlinear dynamical system identification using PID neural networks," International Journal of Nonlinear Sciences and Numerical Simulation, vol. 7, no. 2, pp. 177-182, 2006.

[29] M. M. Mousa and S. F. Ragab, "Application of the homotopy perturbation method to linear and nonlinear schrödinger equations," Zeitschrift fur Naturforschung A, vol. 63, no. 3-4, pp. 140-144, 2008.

[30] J. H. He, "Homotopy perturbation technique," Computer Methods in Applied Mechanics and Engineering, vol. 178, no. 3-4, pp. 257-262, 1999.

[31] J.-H. He, "Homotopy perturbation method: a new nonlinear analytical technique," Applied Mathematics and Computation, vol. 135, no. 1, pp. 73-79, 2003.

[32] J. H. He, "A simple perturbation approach to Blasius equation," Applied Mathematics and Computation, vol. 140, no. 2-3, pp. 217-222, 2003.

[33] J.-H. He, "Homotopy perturbation method for solving boundary value problems," Physics Letters A, vol. 350, no. 12, pp. 87-88, 2006.

[34] A. Golbabai and B. Keramati, "Modified homotopy perturbation method for solving Fredholm integral equations," Chaos, Solitons and Fractals, vol. 37, no. 5, pp. 1528-1537, 2008.

[35] M. Ghasemi, M. T. Kajani, and E. Babolian, "Numerical solutions of the nonlinear Volterra-Fredholm integral equations by using homotopy perturbation method," Applied Mathematics and Computation, vol. 188, no. 1, pp. 446-449, 2007.

[36] J. Biazar and H. Ghazvini, "He's homotopy perturbation method for solving systems of Volterra integral equations of the second kind," Chaos, Solitons and Fractals, vol. 39, no. 2, pp. 770-777, 2009.

[37] Z. Odibat and S. Momani, "A reliable treatment of homotopy perturbation method for Klein-Gordon equations," Physics Letters A, vol. 365, no. 5-6, pp. 351-357, 2007.

[38] M. S. H. Chowdhury and I. Hashim, "Solutions of timedependent Emden-Fowler type equations by homotopyperturbation method," Physics Letters A, vol. 368, no. 3-4, pp. 305-313, 2007.

[39] J. H. He, "Some asymptotic methods for strongly nonlinear equations," International Journal of Modern Physics B, vol. 20, no. 10, pp. 1141-1199, 2006.

[40] A. Meena and L. Rajendran, "Mathematical modeling of amperometric and potentiometric biosensors and system of nonlinear equations-homotopy perturbation approach," Journal of Electroanalytical Chemistry, vol. 644, no. 1, pp. 50-59, 2010.

[41] A. Eswari and L. Rajendran, "Analytical solution of steady state current at a microdisk biosensor," Journal of Electroanalytical Chemistry, vol. 641, no. 1-2, pp. 35-44, 2010. 


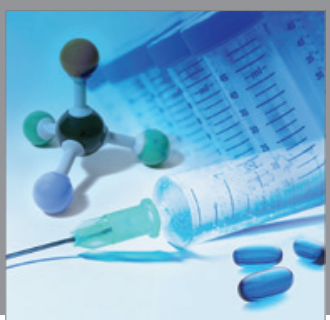

International Journal of

Medicinal Chemistry

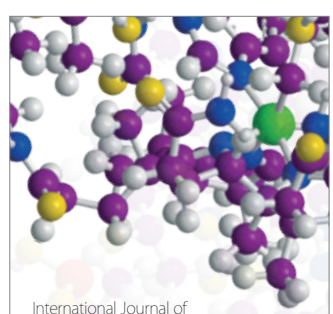

Carbohydrate Chemistry

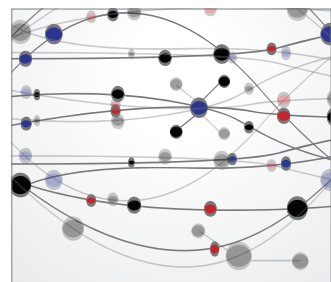

The Scientific World Journal
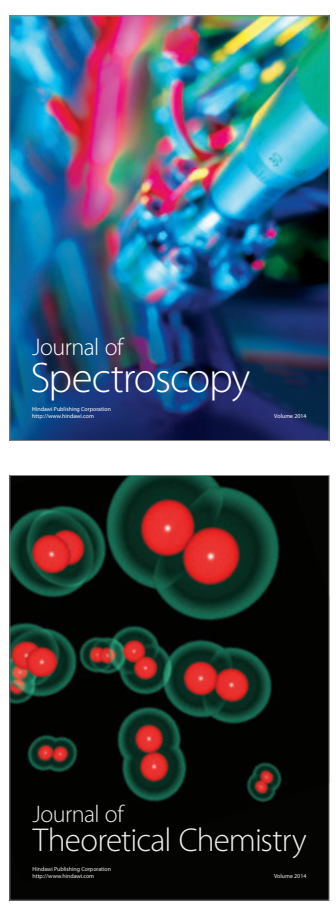
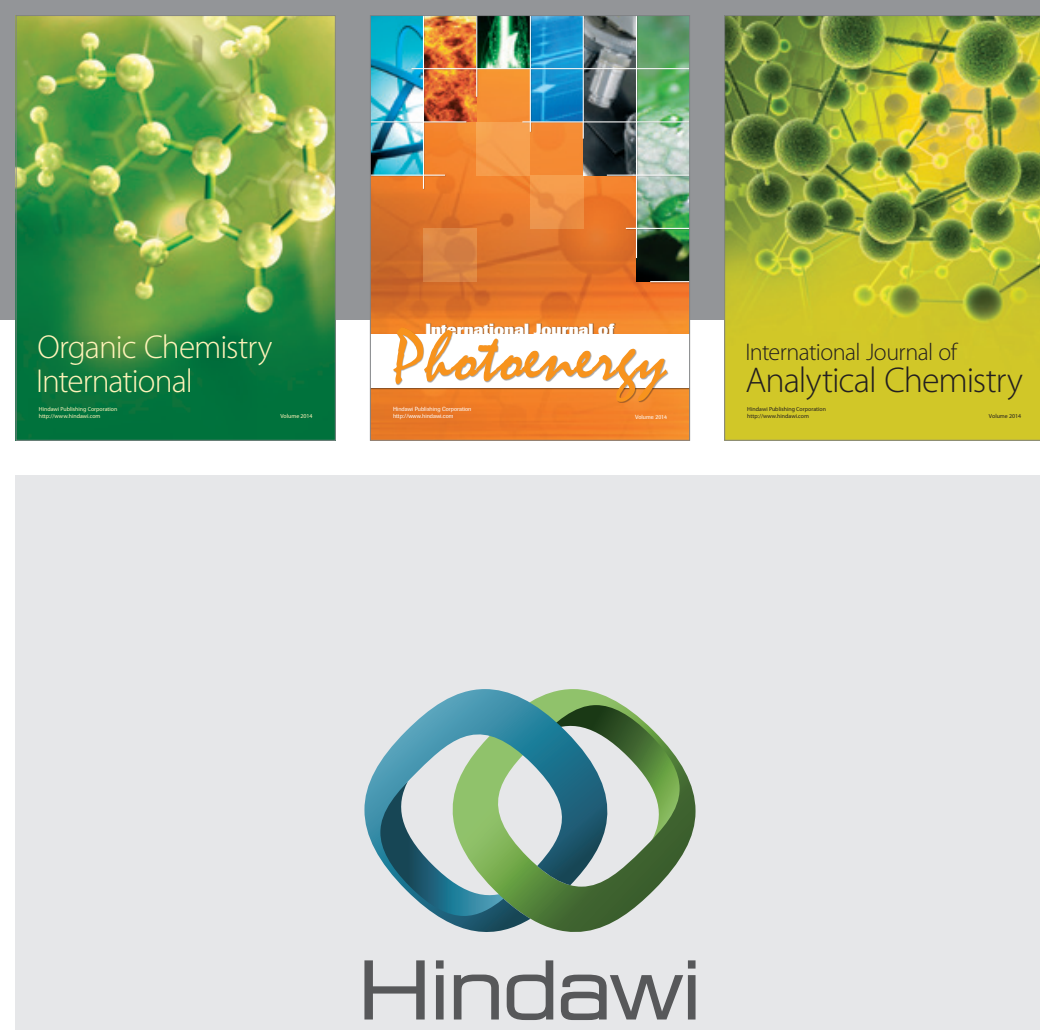

Submit your manuscripts at

http://www.hindawi.com
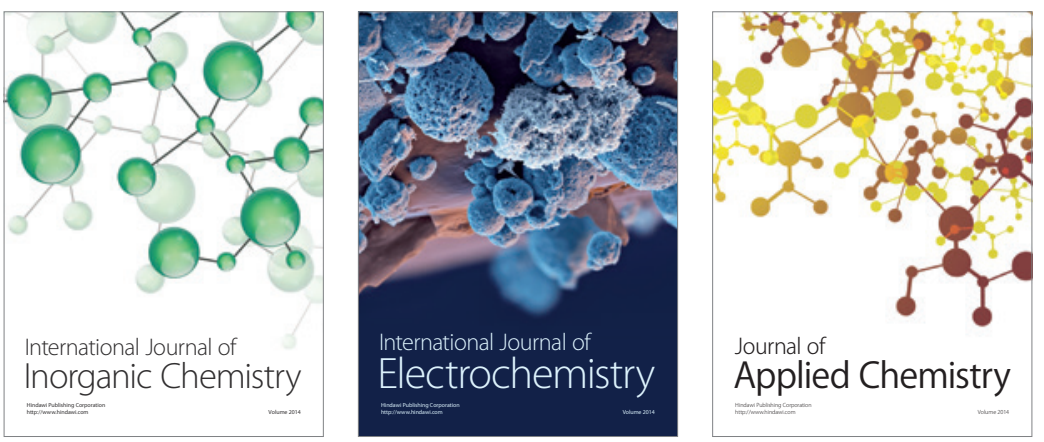

Journal of

Applied Chemistry
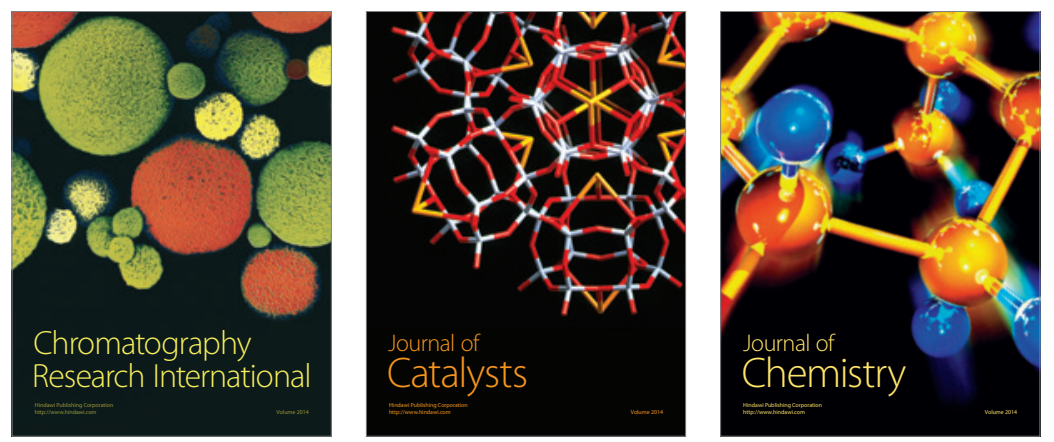
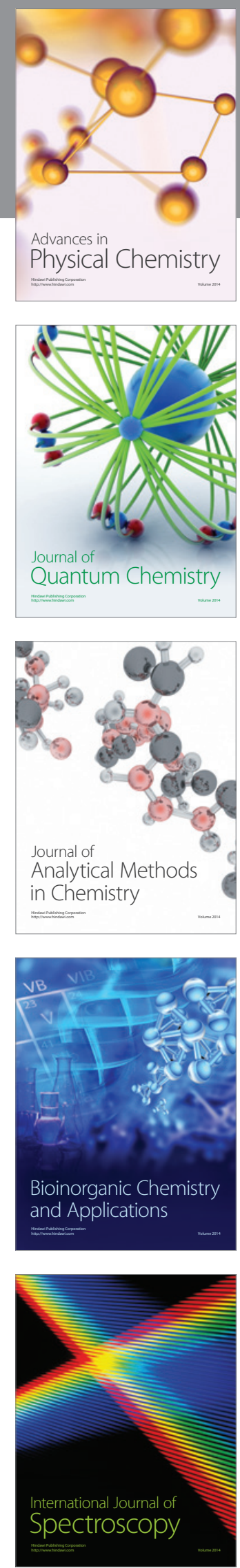